\title{
Safety, efficacy, length of stay and patient satisfaction with outpatient management of low-risk pulmonary embolism patients - a meta-analysis
}

\author{
Aaqib H. Malik, Wilbert S. Aronow
}

Department of Medicine and Cardiology Division, Westchester Medical Center and New York Medical College, Valhalla, NY, USA

Submitted: 14 August 2018

Accepted: 25 October 2018

Arch Med Sci 2021; 17 (1): 245-251

DOI: https://doi.org/10.5114/aoms/99206

Copyright $\odot 2020$ Termedia \& Banach

\section{Abstract}

Introduction: Annual health expense of hospital admissions, due to venous thromboembolism including pulmonary embolism, exceeds 10 billion dollars in the United States. Most of these patients still get admitted to the hospital despite the advent of novel oral anticoagulants. Our aim is to show that lowrisk pulmonary embolism patients can safely be discharged from the emergency department with similar patient satisfaction and lower length of stay. Methods: A comprehensive search in Medline indexed and non-indexed, Embase, and Cochrane Central was performed to search for all the randomized controlled trials that compared inpatient treatment of low-risk pulmonary embolism to outpatient treatment.

Results: Of 68 potentially relevant studies, a total of 2 studies (453 participants) met our inclusion criteria and had data available on patient satisfaction, length of stay, efficacy, and patient safety. The pooled estimate of the included studies showed that at 3-month follow-up, there was no statistically significant difference between inpatient and outpatient treatment of these low-risk patients.

Conclusions: In conclusion, our meta-analysis of 2 randomized controlled trials shows that low-risk pulmonary embolism patients can safely be discharged from the emergency departments in the limited studies available. We need more randomized controlled trials to confirm these findings.

Key words: pulmonary embolism, outpatient treatment, home management, mortality.

Pulmonary embolism (PE) is one of the major health problems with more than 200,000 cases being diagnosed each year in the United States (US) [1]. We have been seeing increasing incidences of PE over the last 10 years due to the increased life expectancy with associated comorbidities in the elderly population, and because of the increased usage of computed tomography (CT) scanning in the emergency departments (ED) [2]. The majority of the economic burden for the management of PE is in the first three days of hospital admission with one study estimating it to be $\$ 6579$ [3], whereas the mean total cost of managing 1 patient with pulmonary embolism was estimated to be $\$ 8764$ [4]. Mortality related to $\mathrm{PE}$ has remained constant despite the improved diagnostic measures [2], and for this reason more than $90 \%$ of ED units in the US admit all the patients with PE [5].

\author{
Corresponding author: \\ Wilbert S. Aronow \\ MD, FACC, FAHA \\ Westchester Medical \\ Center and New York \\ Medical College \\ Macy Pavilion \\ Room 141 \\ Valhalla \\ NY 10595, USA \\ E-mail: wsaronow@aol.com
}


There has been increasing evidence supporting the outpatient management of selected low-risk pulmonary embolism (LRPE) patients. We have had multiple observational studies, prospective studies, and systematic reviews suggesting that the outpatient treatment of LRPE patients is relatively safe. However, ED physicians have been relatively slow in adopting the outpatient management of LRPE. In 2015, Stein et al. found that despite the advent of novel oral anticoagulants (NOAC), $98.3 \%$ of patients with PE are treated as an inpatient [5]. One out of 6 of these admitted patients was eventually discharged within $48 \mathrm{~h}$ of hospitalization. However, an estimated $50 \%$ of these patients could have been discharged potentially safely with an outpatient treatment plan [6]. The concern among physicians is due to lack of evidence from a large randomized trial as well as lack of consensus on criteria to identify these LRPE patients.

Our aim is to summarize the best possible evidence from randomized controlled trials (RCTs) in the form of a meta-analysis to qualitatively and quantitatively analyze the efficacy and safety of the management of these LRPE patients. We also wanted to look at patient satisfaction and length of stay for these patients.

Methods. We searched the following four electronic databases on July 31, 2018: Medline (R), Medline (R) In-Process \& other non-indexed citations, Embase, and Cochrane Central. The keywords 'Pulmonary embolism' and 'outpatient' were indexed in all combinations for original articles and clinical studies. No restrictions for language or publication year were used. Additionally, we manually checked the bibliography of selected original articles and reviews to identify any additional studies.

These articles were evaluated against a priori inclusion and exclusion criteria. Eligible studies: 1) were randomized controlled trials; 2 ) were done in patients with the diagnosis of pulmonary embolism; 3) should involve treatment of pulmonary embolism on an outpatient basis and the patients were discharged from the emergency department, and 4) reported efficacy and safety outcomes along with patient satisfaction data and their length of stay. We excluded studies that, 1) did not evaluate outpatient treatment of PE, 2) had a comparison control group, 3) kept the patients in the ED to form an early discharge group rather than an outpatient group, and 4) collected data separately for PE rather than combining it with deep venous thrombosis.

For each included study, data were collected on the year of publication, participant number and characteristics, duration and frequency of follow-up, mortality, major bleeding, and number of recurrences of venous thromboembolic events (VTE), length of hospital stay and patient satisfaction in both the usual care group and the outpatient treatment group. Quality assessment was performed using the Jadad score [7].

Statistical analysis. The results of each RCT were reported either as a relative risk (RR), hazard ratio, odds ratio (OR), or dichotomous frequency data. We used the frequency of event rates for all studies to compute the cumulative OR and the $p$-values for mortality, length of hospital stays, recurrence of VTE, and major bleeding. P-values $<0.05$ were considered statistically significant. We dichotomized the patient satisfaction data for our analysis. We performed meta-analyses with the DerSimonian and Laird random effects model to obtain the pooled OR for our main outcomes [6]. We used Cochran's Q test to assess heterogeneity between studies and used the $R$ statistic to quantify the proportion of observed inconsistency across study results not explained by chance [7]. All analyses were performed in RevMan version $5.3[8]$.

Results. Our initial search identified 68 publications across the 4 databases. The search of conference proceedings and bibliographies identified 2 additional articles. We narrowed down the potentially relevant unique articles after removing duplicates to 65. After title and abstract screening, we excluded 56 articles for a variety of reasons as mentioned in the PRISMA diagram (Figure 1). We excluded a further 7 articles on full-text review and eventually ended up with 2 reports for our primary analysis for the outcome of mortality, recurrence of VTE, and major bleeding. In addition, we looked at the patient satisfaction, cost of the initial visit and the length of stay.

Table I summarizes the characteristics of the included studies. In the two RCTs, we had 453 patients. Both trials were published within the last 7 years with the first one in 2011 and the latest one in 2018. Both studies scored moderately in quality. There is an inherent bias in a study which differentiates between inpatient versus outpatient as you cannot blind the patients.

The clinical trial Outpatient Treatment of Pulmonary Embolism (OTPE) did have a high exclusion rate [9]. Only $21 \%$ of the total screened patients were included in the primary analysis after randomization. In this study, the time to start anticoagulation in the outpatient group was 16 (5-23) h compared to 8 (3-22) h in the inpatient group, but this difference was statistically insignificant. Hospital readmissions, hospital visits, and home nursing visits were slightly higher in the outpatient group but this difference was also found to be statistically insignificant. As shown in Table I, the authors used the pulmonary embolism severity index (PESI) score to identify low-risk pulmonary embolism patients. 


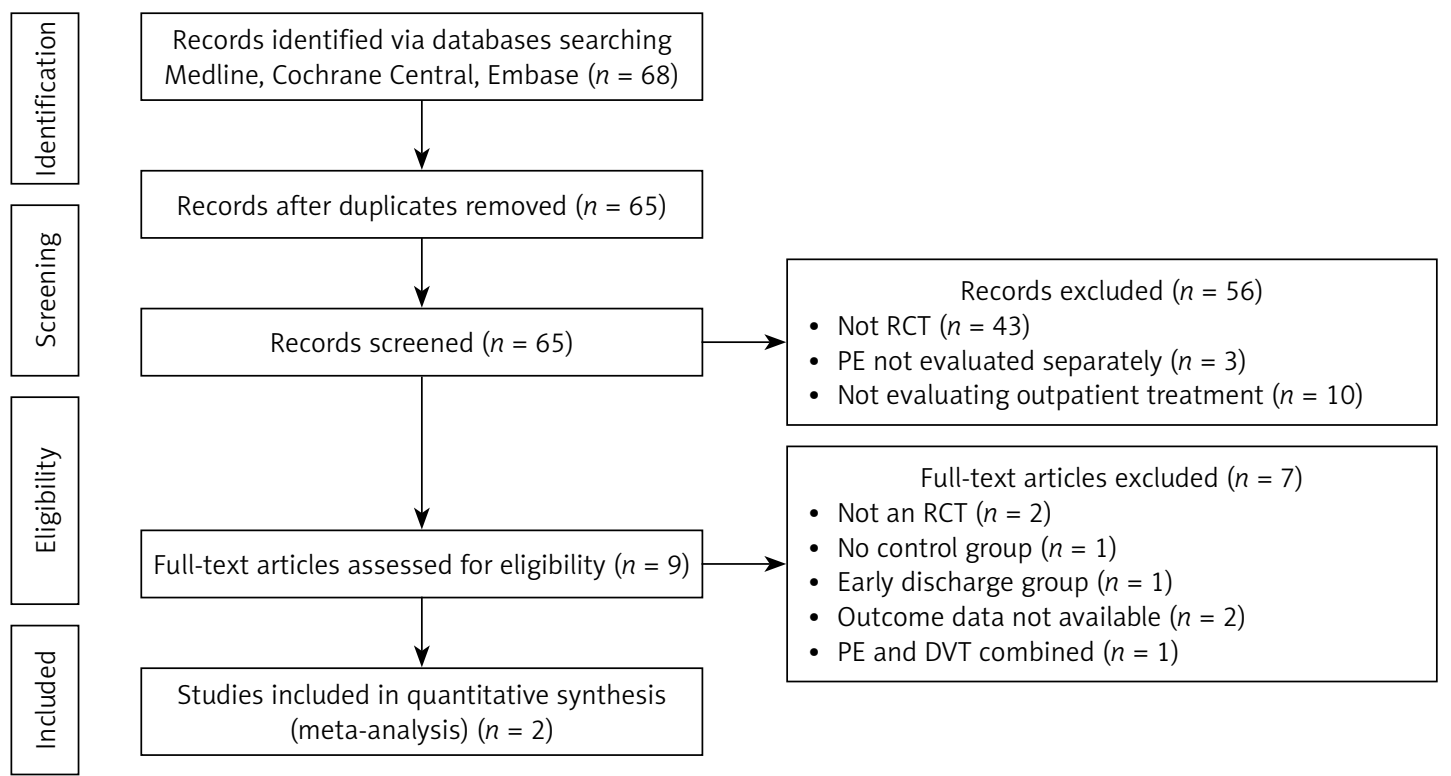

Figure 1. PRISMA diagram for studies selection

$P E$ - pulmonary embolism, DVT - deep vein thrombosis, RCT - randomized controlled trial.

Table I. Baseline characteristics of studies

\begin{tabular}{|lcccccccc|}
\hline Study, year & $\begin{array}{c}\text { Patients } \\
(n)\end{array}$ & $\begin{array}{c}\text { Mean age } \\
\text { [years] }\end{array}$ & $\begin{array}{c}\text { Males } \\
n(\%)\end{array}$ & $\begin{array}{c}\text { Follow-up } \\
\text { [days] }\end{array}$ & $\begin{array}{c}\text { Criteria } \\
\text { used }\end{array}$ & $\begin{array}{c}\text { Previous } \\
\text { VTE \%age }\end{array}$ & $\begin{array}{c}\text { Cancer } \\
\text { patients }(n)\end{array}$ & $\begin{array}{c}\text { Follow-up } \\
\text { loss }(n)\end{array}$ \\
\hline OTPE, 2011 [9] & 339 & 47 & $84(49)$ & 14 and 90 & PESI & $18 \%$ & 1 & 7 \\
\hline $\begin{array}{l}\text { MERCURY PE, } \\
\text { 2018 [10] }\end{array}$ & 114 & 49 & $55(48)$ & 90 & Hestia & $19 \%$ & 7 & 13 \\
\hline
\end{tabular}

OTPE - Outpatient Treatment of Pulmonary Embolism, MERCURY PE - Rivaroxaban for Early Discharge of Low Risk Pulmonary Embolism From the Emergency Department.

The latest study published this year, Rivaroxaban for Early Discharge of Low Risk Pulmonary Embolism From the Emergency Department (MERCURY PE), is the first trial that has utilized novel oral anticoagulants (NOACs) for the outpatient management of PE patients [10]. This study used modified Hestia criteria to identify low-risk PE patients. This study enrolled only $6 \%$ of all the patients screened for low-risk pulmonary embolism. In addition, $5 \%$ of all of the patients were excluded due to noncompliance with medication, and another $8 \%$ of the patients were excluded because the physician or patient did not wish to participate in the study. This trial found that the initial visit cost to the emergency room was $\$ 2,638$ less with outpatient treatment [10]. The total cost was $\$ 2,496$ less with outpatient treatment than with inpatient treatment of these patients.

In the OTPE trial, by post-randomization day 14 , $92 \%$ of the outpatient group patients and $95 \%$ of the inpatient group patients reported being very satisfied or satisfied [9]. In the MERCURY PE study, $60.4 \%$ of the outpatient group and $62.7 \%$ of the inpatient group were very satisfied [10]. Figure 2 shows a forest plot for patient satisfaction.
In the OTPE trial, the length of initial hospital stay for the outpatient and inpatient groups was 12 and $93.6 \mathrm{~h}$, respectively [9]. In the MERCURY PE study, the length of stay was $19.2 \mathrm{~h}$ in the outpatient group versus $43.2 \mathrm{~h}$ in the inpatient group [10]. Figure 3 shows a forest plot for the length of stay.

In the OTPE study, there was only 1 patient who had recurrent VTE in the outpatient treatment group. This patient had cervical cancer, and recurrent VTE happened on day 83 [9]. The MERCURY PE trial did not show any recurrence of VTE during the 3 months after randomization in both groups [10]. Figure 4 shows a forest plot for recurrent VTE.

In the OTPE study, the only death in the outpatient group was not related to PE. The patient died from an accident-related trauma and aortic rupture. There was 1 death in the inpatient group, a patient who died of lung cancer and pneumonia [9]. In the MERCURY PE trial, no deaths occurred in either the outpatient or inpatient groups [10] Figure 5 shows a forest plot for mortality.

In the OTPE trial, 3 patients had major bleeding in the outpatient group, with 2 patients having intramuscularbleeding on day 3 and day 13 , and the third patient having menometrorrhagia on day 50 [9]. 
No patient had major bleeding in the inpatient group. In the MERCURY PE study, no patient in the inpatient or outpatient group had major bleeding [10]. Table II shows the exclusion criteria used by both randomized controlled trials [9, 10]. Figure 6 shows a forest plot for major bleeding.

Discussion. In this meta-analysis, we assessed the evidence from two RCTs regarding homebased outpatient treatment of low-risk pulmonary PE patients. Our study also shows that patient satisfaction and quality of life remain favorable regardless of the inpatient or outpatient treatment of these LRPE patients. Bledsoe et al. also reported in August 2018 that patient satisfaction was high (91\%) in the outpatient management of these patients [1].
Outpatient management of these LRPE patients results in a shorter hospital stay leading to decreased healthcare costs and less nosocomial infections with an improved quality of life [11-13] Our analysis of outcomes that include mortality, recurrence of VTE and major bleeding shows that outpatient treatment of LRPE patients is effective as well as safe. These findings are consistent with previous prospective and observational studies [14-26].

Our meta-analysis adds to the growing body of data supporting the case for discharging LRPE patients from the emergency department with an outpatient treatment plan. The trials in our study were performed in a heterogeneous group of patients in a diverse healthcare setting in different

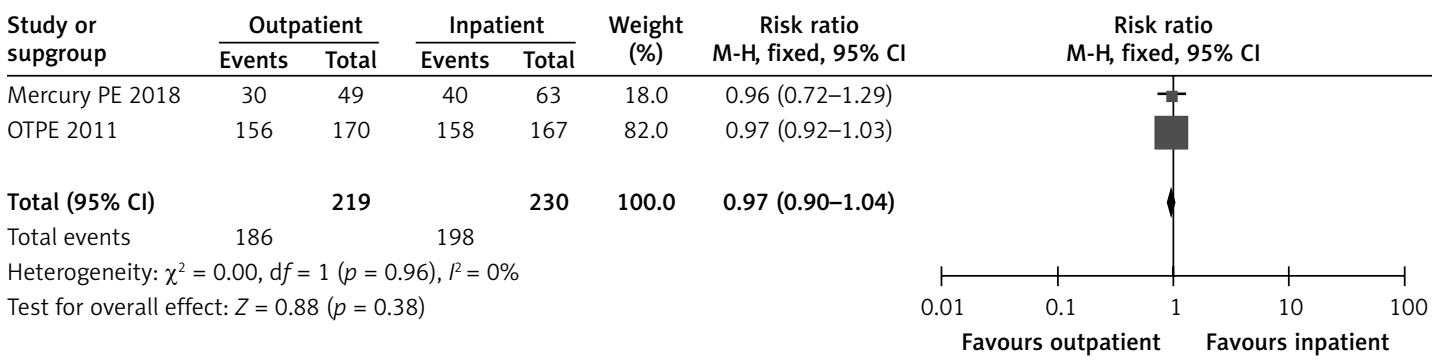

Figure 2. Forest plot: patient satisfaction

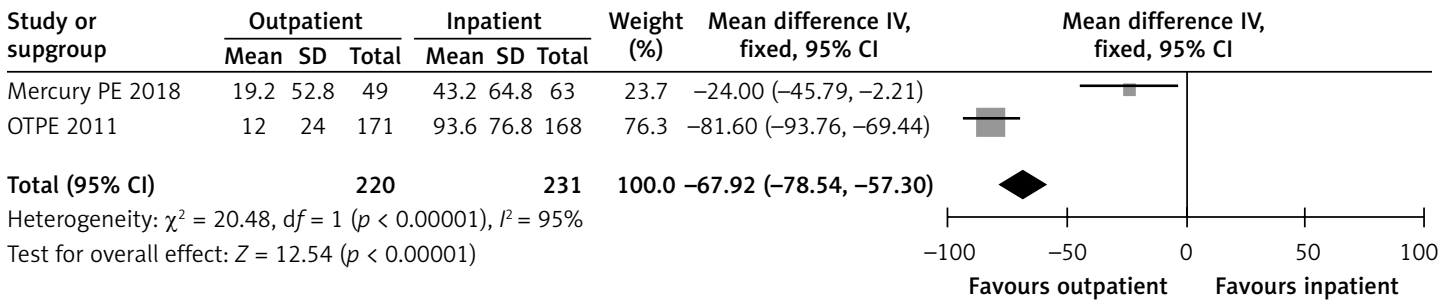

Figure 3. Forest plot: length of stay in hours

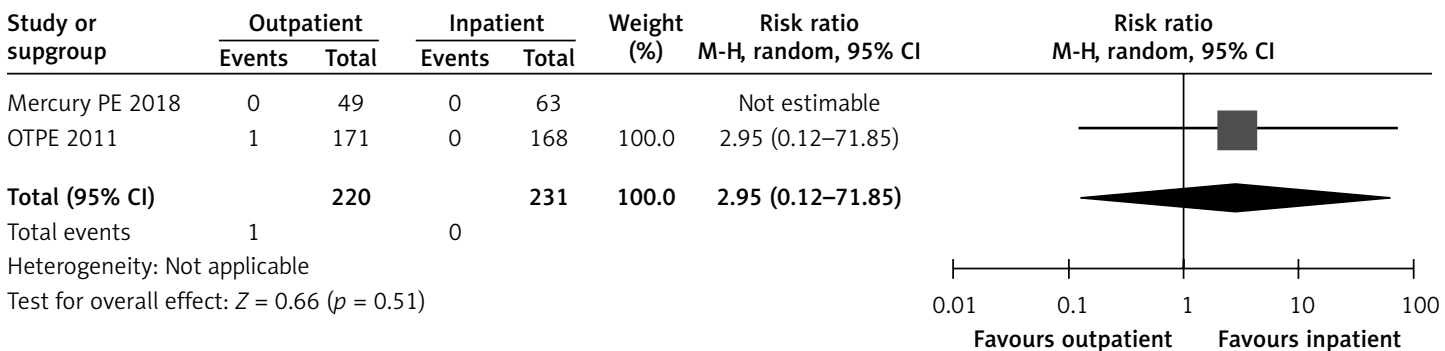

Figure 4. Forest plot: recurrent venous thromboembolism (VTE)

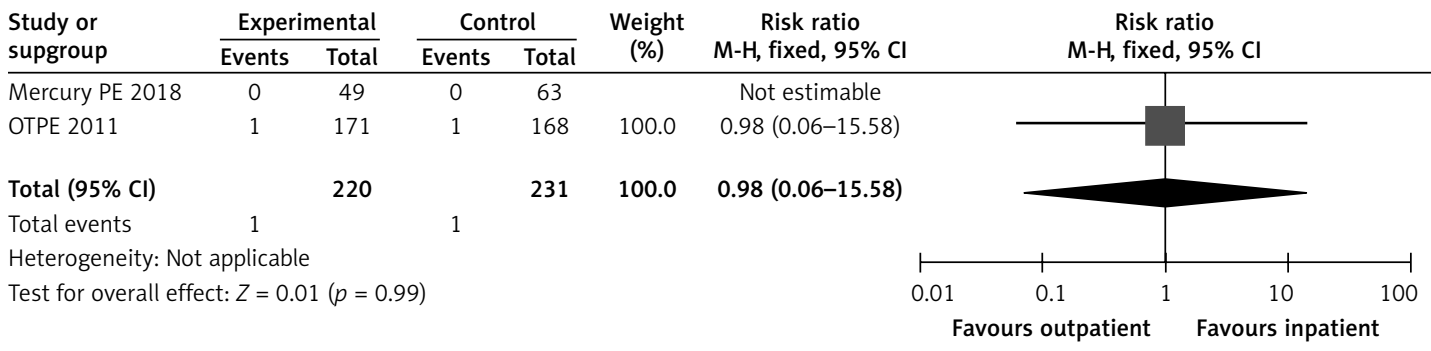

Figure 5. Forest plot: mortality 
Table II. Exclusion criteria used for low risk pulmonary embolism patients in both trials

\begin{tabular}{|lc|}
\hline Did not meet inclusion criteria & Were therapeutic on oral anticoagulation \\
\hline High-risk PE patients & Systolic $\mathrm{BP}<100 \mathrm{~mm} \mathrm{Hg}$ \\
\hline Not objectively confirmed PE & Pregnancy \\
\hline$<18$ years of age & Severe renal failure \\
\hline PE diagnosed after $23 \mathrm{~h}$ & Were previously enrolled in the trial \\
\hline Barriers to adherence/follow-up or non-compliance & Cancer diagnosis \\
\hline Chest pain necessitating parenteral opioids & Troponin elevation \\
\hline Could not consent/declined consent & Physicians' discomfort to enroll \\
\hline Hypoxemic & Medication contraindicated \\
\hline Had active bleeding or high risk of bleeding & \\
\hline
\end{tabular}

countries including Switzerland, France, Belgium, and the United States. We specifically excluded the study by Otero et al. from our analysis since it compared the patients discharged after the third day (early discharge) to routine discharge [14]. Due to the lack of a control group, we also excluded the study by den Exter et al. [15] In the United States, most patients with PE are admitted to the hospital regardless of their risk status. This is partly due to a lack of a standardized widely accepted risk stratifying system as well as physicians having discomfort in discharging a patient with a disease which has a 30-day mortality rate approaching $30 \%$ in some cases [27]. The treatment of VTE is extremely variable and is highly contingent upon the physicians' personal choice, resources available, insurance coverage, and the type of clinical setting [28].

Outpatient management of LRPE patients comes with the caveat that there should be an appropriate and safe outpatient follow-up. In addition, the patients need to be counseled in the emergency department regarding their disease risk and the importance of adherence to their medication. This transition of care (TOC) is critical in the outpatient management of LRPE patients as the gaps in the TOC can often lead to adverse outcomes with a resultant increased mortality and morbidity. The outpatient management of LRPE patients has further been aided with the emergence and widespread availability of NOACs. These newer medications do not need closer monitoring and have the benefit of being effective immediately without any overlap requirement with heparin and for two of them (apixaban and rivaroxaban) without a need of heparin pre-treatments. The TOC is much more simplified, and there are no dietary restrictions. Unfortunately, the adaptation of these newer NOACs has been variable, with one study showing that only $33 \%$ of the patients with PE are being discharged on NOAC [5], even though in 2016 the American College of Chest Physicians (ACCP) gave NOACs priority over vitamin $\mathrm{K}$ antagonists in PE treatment [29]. The same document upgraded the recommendation for outpatient management of LRPE to grade 2B [29].

The major hurdle in adopting the outpatient management of LRPE is the lack of a standardized robust scoring system to identify LRPE patients. There are several scoring systems that have been devised and validated to answer that question. The Hestia criteria and PESI score have been most validated. Barrios et al. found in the PROTECT (Prognostic Value of CT Scan in Hemodynamically Stable Patients with Acute Symptomatic PE) trial a $100 \%$ negative predictive value for all-cause mortality by using the simplified PESI score with multidetector computed tomography (MDCT) to evaluate for right heart strain [30]. Currently MDCT is widely used to assess the severity of PE because

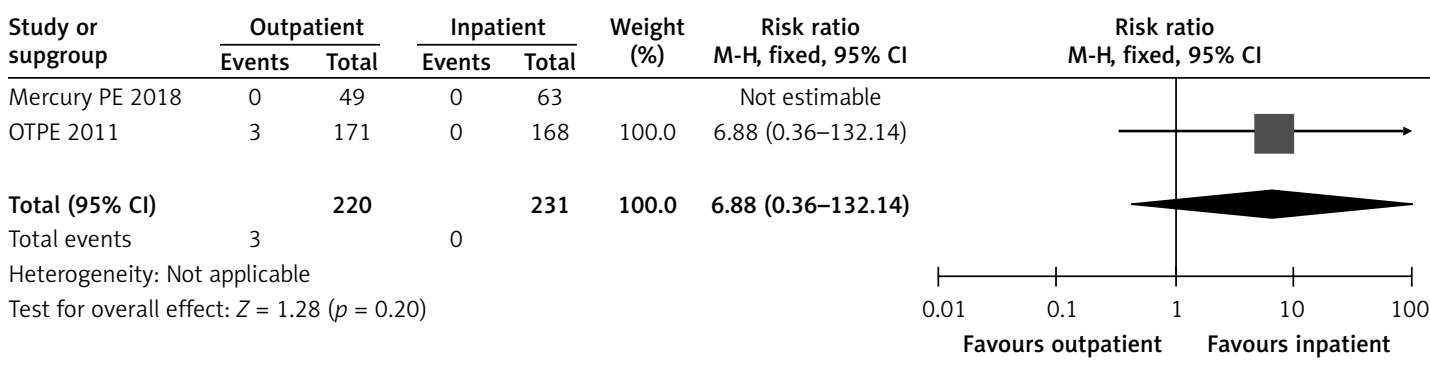

Figure 6. Forest plot: major bleeding 
of its widespread availability [31]. Several other methods can be used to assess the fluid overload often seen in right heart failure patients with high risk $P E$, and that may include incorporating $\mathrm{N}$-terminal pro-B-type natriuretic peptide [32]. The perfect scoring system must be a combination of clinical, biomarker, radiological, and social factors as any single measure such as oxygen saturation has proven to be unreliable in the past [33].

Our meta-analysis has several limitations. First, the number of included studies is small. We need data from more randomized controlled trials. Secondly, the choice of anticoagulation is not standardized. We might be able to have a consistent approach and more reproducible results with an increased utilization of NOACs.

In conclusion, our comprehensive analysis suggests that outpatient treatment of LRPE is feasible, safe, effective, and preferred by the patients. Future studies such as the Hospitalization or Out-treatment Management of Patients with Pulmonary Embolism (HOME-PE) randomized controlled trial NCT02811237 should further clarify the treatment of LRPE patients. We conclude that after careful risk stratification, LRPE patients should be considered for discharge from the emergency department. This would result in a reduction in cost, improved patient satisfaction, and quality of life, and decreased nosocomial complications without any negative effects on mortality or morbidity.

\section{Conflict of interest}

The authors declare no conflict of interest.

\section{References}

1. Bledsoe RJ, Woller CS, Stevens MS, et al. Management of low-risk pulmonary embolism patients without hospitalization: The Low-Risk Pulmonary Embolism Prospective Management Study. Chest 2018; 154: 249-56.

2. Burge JA, Freeman DK, Klapper JP, Haramati BL Jr. Increased diagnosis of pulmonary embolism without a corresponding decline in mortality during the $\mathrm{CT}$ era. Clin Radiol 2008; 63: 381-6.

3. Dasta FJ, Pilon D, Mody HS, et al. Daily hospitalization costs in patients with deep vein thrombosis or pulmonary embolism treated with anticoagulant therapy. Thromb Res 2015; 135: 303-10.

4. Fanikos J, Rao A, Seger CA, et al. Hospital costs of acute pulmonary embolism. Am J Med 2013; 126: 127-32.

5. Stein DP, Matta F, Hughes GP, et al. Home treatment of pulmonary embolism in the era of novel oral anticoagulants. Am J Med 2016; 129: 974-7.

6. Jadad RA, Moore AR, Carroll D, et al. Assessing the quality of reports of randomized clinical trials: is blinding necessary? Control Clin Trials 1994; 17: 1-12.

7. Jackson D, Bowden J, Baker R. How does the DerSimonian and Laird procedure for random effects meta-analysis compare with its more efficient but harder to compute counterparts? J Stat Plan Infer 2010; 140: 961-70.
8. Collaboration C. Review Manager (RevMan) [Computer Program] Version 5.2. 3. Copenhagen: The Nordic Cochrane Centre 2012

9. Aujesky D, Roy PM, Verschuren F, et al. Outpatient versus inpatient treatment for patients with acute pulmonary embolism: an international, open-label, randomised, noninferiority trial. Lancet 2011; 378: 41-8.

10. Peacock WF, Coleman Cl, Diercks DB, et al. Emergency department discharge of pulmonary embolus patients. Acad Emerg Med 2018; 25: 995-1003.

11. Aujesky D, Mazzolai L, Hugli O, Perrier A. Outpatient treatment of pulmonary embolism. Swiss Med Wkly 2009; 139: 685-90.

12. Aujesky D. Cost-effectiveness of low-molecular-weight heparin for treatment of pulmonary embolism Chest 2005; 128: 1601-10.

13. Shepperd S, Doll H, Angus MR, et al. Admission avoidance hospital at home. Cochrane DB Syst Rev 2008; 4: CD007491.

14. Otero R, Uresandi F, Jiménez $D$, et al. Home treatment in pulmonary embolism. Thromb Res 2010; 126: e1-5.

15. den Exter LP, Zondag W, Klok AF, et al. Efficacy and safety of outpatient treatment based on the Hestia clinical decision rule with or without N-terminal pro-brain natriuretic peptide testing in patients with acute pulmonary embolism. A randomized clinical trial. Am J Resp Crit Care 2016; 194: 998-1006.

16. Kovacs JM, Anderson D, Morrow B, et al. Outpatient treatment of pulmonary embolism with dalteparin. Thromb Haemostasis 2000; 83: 209-11.

17. Beer HJ, Burger M, Gretener S, et al. Outpatient treatment of pulmonary embolism is feasible and safe in a substantial proportion of patients. J Thromb Haemost 2003; 1: 186-7.

18. Wells SP, Anderson RD, Rodger AM. A randomized trial comparing 2 low-molecular weight heparins for the outpatient treatment of deep vein thrombosis and pulmonary embolism. Arch Intern Med 2005; 165: 733-8.

19. Olsson CG, Bitzén U, Olsson B, et al. Outpatient tinzaparin therapy in pulmonary embolism quantified with ventilation/perfusion scintigraphy. Med Sci Monitor 2006; 12: PI9-3.

20. Erkens GPM, Gandara E, Wells P, et al. Safety of outpatient treatment in acute pulmonary embolism. J Thromb Haemost 2010; 8: 2412-7.

21. Kovacs JM, Hawel DJ, Rekman FJ, Lazo-Langner A. Ambulatory management of pulmonary embolism: a pragmatic evaluation. J Thromb Haemost 2010; 8: 2406-11.

22. Ong SB, Karr AM, Chan YDK, et al. Management of pulmonary embolism in the home. Med J Australia 2005; 183: 239-42.

23. Zondag W, Mos MIC, Creemers-Schild D, et al. Outpatient treatment in patients with acute pulmonary embolism: the Hestia Study. J Thromb Haemost 2011; 9: 1500-7.

24. Zondag W, den Exter LP, Crobach TMJ, et al. Comparison of two methods for selection of out of hospital treatment in patients with acute pulmonary embolism. Thromb Haemostasis 2013; 109: 47-52.

25. Davies HCW, Wimperis J, Green SE, et al. Early discharge of patients with pulmonary embolism: a two-phase observational study. Eur Respir J 2007; 30: 708-14.

26. Agterof JM, Schutgens GRE, Snijder JR, et al. Out of hospital treatment of acute pulmonary embolism in patients with a low NT-proBNP level. J Thromb Haemost 2010; 8: 1235-41. 
27. Bĕlohlávek J, Dytrych V, Linhart A. Pulmonary embolism, part I: epidemiology, risk factors and risk stratification, pathophysiology, clinical presentation, diagnosis and nonthrombotic pulmonary embolism. Exp Clin Cardiol 2011; 18: 129-38.

28. Guyatt HG, Norris LS, Schulman S, et al. Methodology for the development of antithrombotic therapy and prevention of thrombosis guidelines: antithrombotic therapy and prevention of thrombosis, 9th ed: American College of Chest Physicians Evidence-Based Clinical Practice Guidelines. Chest 2012; 141 (2 Suppl): 53S-70S.

29. Kearon C, Akl AE, Ornelas J, et al. Antithrombotic therapy for VTE disease: CHEST Guideline and Expert Panel Report. Chest 2016; 149: L315-52.

30. Barrios D, Morillo R, Lobo LJ, et al. Assessment of right ventricular function in acute pulmonary embolism. Am Heart J 2017; 185: 123-9.

31. Paczyńska M, Sobieraj P, Burzyński Ł, et al. Tricuspid annulus plane systolic excursion (TAPSE) has superior predictive value compared to right ventricular to left ventricular ratio in normotensive patients with acute pulmonary embolism. Arch Med Sci 2016; 12: 1008-14.

32. Siriopol I, Siriopol D, Voroneanu L et al. Predictive abilities of baseline measurements of fluid overload, assessed by bioimpedance spectroscopy and serum $\mathrm{N}$-terminal pro-B-type natriuretic peptide, for mortality in hemodialysis patients. Arch Med Sci 2017; 13: 1121-9.

33. Erol S, Gürün AK, Arslan FC, et al. Is oxygen saturation variable of simplified pulmonary embolism severity index reliable for identification of patients, suitable for outpatient treatment? Clin Respir J 2018; 12: 762-6. 